\title{
2 AGUA YTERRITORIO \\ Derecho humano al agua potable en la localidad de Tlachichilco del Carmen en el municipio de Poncitlán, Jalisco, México: análisis preliminar de un problema en un territorio periurbano
}

\author{
Human rights to water in Tlachichilco del Carmen, Poncitlán, Jalisco, \\ Mexico: preliminary analysis of a problem in a periurban territory
}

Francisco Jalomo-Aguirre Universidad de Guadalajara Guadalajara, México jalomo19@hotmail.com

Alicia Torres-Rodríguez Universidad de Guadalajara Guadalajara, México atorres59@gmail.com

Leonor Ceballos-González Comisión Nacional Forestal Zapopan, México leonorceballosg@hotmail.com

Juan Pablo Avila-De Alba Universidad de Guadalajara Guadalajara, México pablo.aviladealba@gmail.com

Lorena Tanit Álvarez-Cortázar Universidad de Guadalajara Guadalajara, México lorena_tanit21@hotmail.com

Resumen - A pesar de ser el agua un recurso vital para la existencia de cada especie del planeta Tierra; pese a haber sido reconocido, en declaraciones internacionales de diversos tipos, como un derecho de todos los individuos, tal recurso no se encuentra distribuido y disponible en igualdad, cantidad y calidad, por tanto existe hoy en día una creciente problemática por asegurar tal garantía, objetivo a demostrar en el presente documento, que muestra los resultados de un estudio realizado en la localidad de Tlachichilco del Carmen, en el municipio de Poncitlán, Jalisco, México.

Así, mediante una revisión exhaustiva de fuentes documentales y aplicación de entrevistas y encuestas, se evidencia en este texto el problema de acceso al agua y las estrategias, como la captación de agua de lluvia que están sucediendo en una localidad conlindante al cuerpo de agua dulce más grande de México, el lago de Chapala, ubicado en el occidente de México.

Abstract - The Water is a vital resource for the existence of every species of planet Earth; Despite having been recognized in international declarations of various types as a right of all individuals, such a resource is not distributed and available in equality, quantity and quality, so there is now a growing problem to ensure such a guarantee, Objective to demonstrate in the present document, which shows the results of a study carried out in the town of Tlachichilco del Carmen, in the municipality of Poncitlán, Jalisco, Mexico.

Thus, through an exhaustive review of documentary sources and the application of interviews and surveys, the text of this paper shows the problem of access to water and strategies, such as rainwater harvesting that are happening in a locality linked to the freshwater body Largest in Mexico.

Palabras clave: Derecho humano al agua, Tlachichilco, Territorio periurbano, Guerra por el agua

Keywords: Human rigths to water, Tlachichilco, Periurban territory, War for water 


\section{INTRODUCCIÓN}

Desde la década de los sesenta se planteó como centro de discusiones internacionales el desarrollo sustentable, con la publicación de la obra Silent Spring. Desde entonces, tal asunto, ha sido la respuesta, que a decir de diversos pensadores sociales como Baudrillard y Vattimo $^{1}$, atiende los efectos negativos de la modernidad, la creciente individualidad contemporánea, la pérdida de lo colectivo, el resquebrajamiento del imaginario social, la era del vacío y la sociedad del consumo. Aunque la idea del desarrollo sustentable ha sido usada desde diferentes visiones, debe ser vista como un futuro común a todas las especies, que permitiría satisfacer los requisitos del presente, sin comprometer la capacidad de generaciones futuras $\mathrm{y}$ ancestrales para satisfacer sus propias necesidades ${ }^{2}$, acuñando así el concepto de desarrollo sustentable que sigue vigente hasta nuestros días.

El desarrollo sustentable es entonces aquel que busca un equilibrio entre las exigencias del mercado, la sociedad y el entorno natural o ecológico. Efectivamente, el centro de equilibrio es el desarrollo sustentable ${ }^{3}$, que busca la armonía entre las cuatro dimensiones y/o esferas en que podemos clasificar el medio ambiente: la natural o ecológica, la económica representada por el mercado, la social y la ética y cultural.

Bajo la noción antes planteada, es adecuado afirmar que la gestión del agua dulce en el mundo actual no cuenta con mecanismos que garanticen su sustentabilidad. $^{4}$ Por lo tanto, la realización del consecuente derecho humano al agua, tal como se evidenciará en el estudio en curso, no ocurre en la localidad de Tlachichilco del Carmen, en pleno siglo XXI. Es claro, el agua es un recurso vital para la existencia, base fundamental para la sustentabilidad, pero no es accesible en igualdad, cantidad y calidad para todos. Aunque ha sido reconocido en declaraciones internacionales de diversos tipos el acceso al agua como un derecho para todos los hombres, en pleno siglo XXI existe una creciente problemática por garantizar su realización.

Si bien la mayoría de discusiones al respecto aseveran que son las zonas urbanas las que acaparan el recurso, el presente análisis pretende abrir la puerta para discusiones futuras: los resultados obtenidos con trabajo de campo muestran que al interior de los propios espacios periurbanos (entendiéndose como espacios rurales) comienzan también a evidenciar problemáticas al respecto. El documento presente demuestra, con un planteamiento metodológico aplicado a un área de estudio específico, la existencia de un problema creciente, actual, con el agua. Elemento que sufre entre las pugnas del mercado, el poder, dominación y mercantilización ${ }^{5}$. Pugnas que dificultan materializar el derecho humano al agua para todos, en igualdad de condiciones.

\footnotetext{
1 Baudrillard, 1970 y Vattimo, 1981.

${ }^{2}$ Fernández, 2000, negritas propias.

${ }^{3}$ Allen, 1994.

4 Jalomo, 2016

5 García, 2006.
}

\section{PlanTEAMIENTO METOdOLÓGiCo}

Para realizar el análisis se revisaron fuentes documentales que permitieron reconstruir la historia de Tlachichilco del Carmen. Se revisó literatura relacionada al derecho humano al agua, sustentabilidad y territorio periurbano. Con ello, se construyó una base teórica que permitió recoger información, mediante encuestas y entrevistas en campo, que posibilitaron interpretaciones preliminares sobre el problema presente en la localidad periurbana antes mencionada. Una problemática relacionada con accesibilidad a tal recurso natural, elemental para la vida, y que genera procesos de tensión social $^{6}$ en diversos colectivos sociales.

En trabajo de campo, se aplicaron 206 encuestas y 4 entrevistas. Cumpliendo, en el caso de las primeras, con el método recomendado por Suárez ${ }^{7}$, efectivo para calcular el tamaño de la muestra y que la información recolectada, fuese representativa del universo abordado.

Así, a fin de conocer el tamaño de la muestra, se usó la siguiente fórmula:

$$
n=\frac{N \sigma^{2} Z^{2}}{(N-1) e^{2}+\sigma^{2} Z^{2}}
$$

Formula en donde $n$ representa el tamaño de la muestra seleccionada para el estudio; $\mathrm{N}$ representa el tamaño de la población abordada, es igual a la desviación estándar de la población que, generalmente tiene un valor constante de 0,5 ; por su parte, $Z$ representa el valor o nivel de confianza; e representa el límite aceptable de error muestral que, para efectos de este estudio, fue considerado entre el $1 \%(0,01)$ y $9 \%(0,09)$. Por lo anterior, se calculó el tamaño de la muestra para una población de 437 domicilios con un total muestral de 135 domicilios -que equivale un 31\% de la población total-, mismos que representan un nivel de confianza del 95\%.

La aplicación de formatos de encuestas a la población seleccionada y los formatos de entrevistas a los actores clave del territorio, sirvieron para alimentar la base de datos. La recolección de datos y aplicación de los formatos se realizó gracias al apoyo voluntario de estudiantes de la Licenciatura en Geografía y Ordenación Territorial del Centro Universitario de Ciencias Sociales y Humanidades (CUCSH), como parte de las actividades de campo de la asignatura Métodos de Investigación en Ciencias Sociales, durante el año 2016.

Entre los actores clave entrevistados para recabar la información aquí estudiada se encuentran:

- El Agente Municipal en Tlachichilco del Carmen, de apeído Betancourt, hermano de...

- El Auxiliar de Bombas, Salvador Betancourt.

- María Elena Muñoz, vecina de la localidad y encargada de educación del programa PROSPERA.

- Mtra. Leonor Ceballos González, posesionaria de un terreno y actor social incansable.

Quedaron pendientes entrevistas a otros actores claves: el Presidente Municipal de Poncitlán, Juan Carlos Montes Johnston y al Jefe del Departamento de Agua Potable y Obras Públicas del mismo municipio. A su vez,

\footnotetext{
6 Gómez, 2012.

${ }^{7}$ Suarez, 2004.
} 
aplicar encuestas a ciudadanos canadienses y norteamericanos, también parte del problema. Objetivo por cumplir en una segunda etapa de la investigación. Se llevó a cabo una selección de diversas técnicas tanto cualitativas, como cuantitativas, que permitieron acceder a la mayor información representativa de la realidad y de retos y problemas relacionados al acceso equitativo al agua.

Uno de los informantes ya mencionado, María Elena Muñoz, fue factor clave para lograr introducirse en la localidad, no es fácil ni adecuado llegar como extraños a tocar puertas y hacer preguntas; ella fue acceso primordial para aplicar un pequeño cuestionario sobre el hogar a jefes y jefas de cada domicilio: cuántos integrantes lo conformaban, cuánto tiempo tenían viviendo en Tlachichilco, qué uso le daban a la escasa agua recibida al día. Así se logró captar un panorama general sobre el uso del recurso y su administración en la localidad, con el propósito de analizar y enfrentar la serie de problemáticas de dicho territorio periurbano. Conocer cómo encaran su vida diaria, al no disponer del vital líquido en forma accesible, asequible, bajo criterios de no discriminación y en calidad suficiente para satisfacer sus necesidades individuales y colectivas, fue un objetivo alcanzado de bastante importancia.

La investigación no solamente se acotaba a conocer con cuánta agua se disponía o cuánto tiempo la recibían en cada domicilio, o si era de calidad o no y cuánto pagan por ella; a la par, se quiso comprender las raíces más profundas que subyacen a la problemática. Con las técnicas e instrumentos utilizados en trabajo de campo, fue posible entender los sacrificios que conlleva vivir sin agua en acciones tan comunes como tomar un baño o lavar la ropa, exponiendo los problemas sociales y las tensiones emanados de una comunidad. En conjunto, lo anterior funcionó para descubrir una problemática aún mayor y conocer las acciones emprendidas día a día por revertir tal situación.

El formato de encuesta estaba pensado de forma tal, que fuera fácil de entender tanto para los encuestadores como para los encuestados, y estructurado con 20 preguntas dirigidas a comprender el problema de sustentabilidad y derecho humano al agua potable. El trabajo de campo se realizó dividiendo al grupo de encuestadores de forma aleatoria con la

Fuente: Elaboración propia.

Fuente: Elaboración propia. intensión de obtener información diversa, en diferentes cuadrantes de la comunidad. De tal forma, se posibilitó que al sistematizar la información, comparar resultados y someter los datos cuantitativos a diferentes análisis multivalentes, se comprobara mediante cifras un sin número de contrariedades y grandes dificultades que viven los lugareños cotidianamente, que se muestran en la parte final de este documento.

UBICACIÓN Y ANTECEDENTES HISTÓRICOS DE UNA LOCALIDAD EN PUGNA

La Comunidad Indígena de Tlachichilco del Carmen, se localiza al suroeste del municipio de Poncitlán en el Estado de Jalisco y colinda con el Lago de Chapala ${ }^{8}$, que es el cuerpo de agua dulce más grande de México, tal y como se muestra en los mapas 1 y 2 respectivamente. Se sitúa a 1.530 metros de altitud sobre el nivel del mar y sus coordenadas geográficas son: longitud $20^{\circ} 20^{\prime} 00^{\prime \prime}$ y latitud $103^{\circ} 05^{\prime} 20^{\prime \prime 9}$. Es un asentamiento humano que desde 1547 pertenecía a la Comunidad Indígena de San Juan Tecomatlán bajo la categoría de barrio, pero mediante un proceso jurídico de implicaciones sociales, los habitantes se dividieron para adquirir identidad propia y sentido de pertenencia, así el 25 de julio del 2012 se separa de San Juan Tecomatlán para formar una nueva comunidad denominada "Comunidad Indígena de Tlachichilco del Carmen” ${ }^{10}$.

Mapa 1 .Ubicación de la Comunidad Indígena y/o localidad de Tlachichilco del Carmen

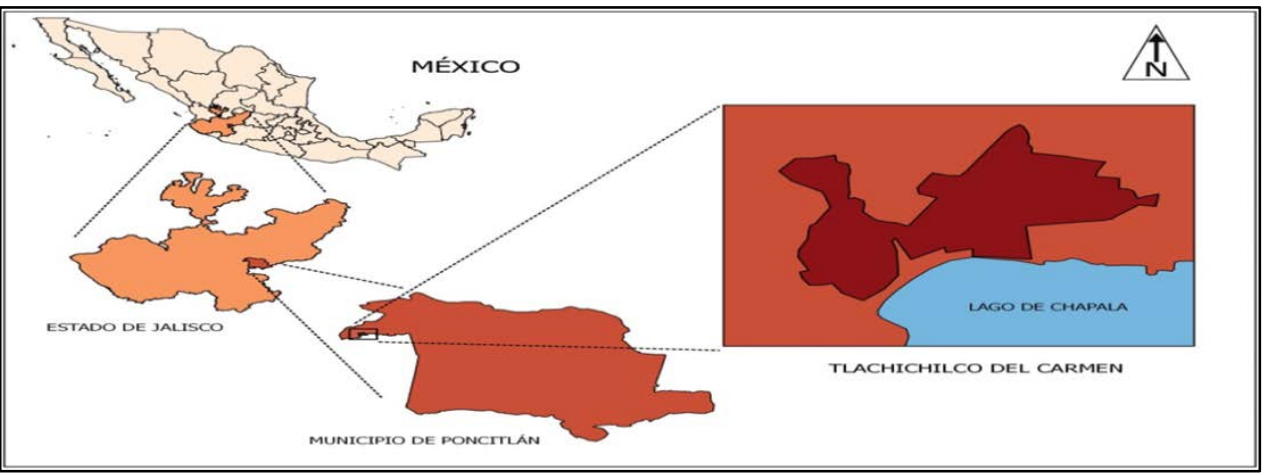

Mapa 2. Comunidad Indígena y/o localidad de Tlachichilco del Carmen en relación con el Lago de Chapala y la localidad de San Juan Tecomatlán

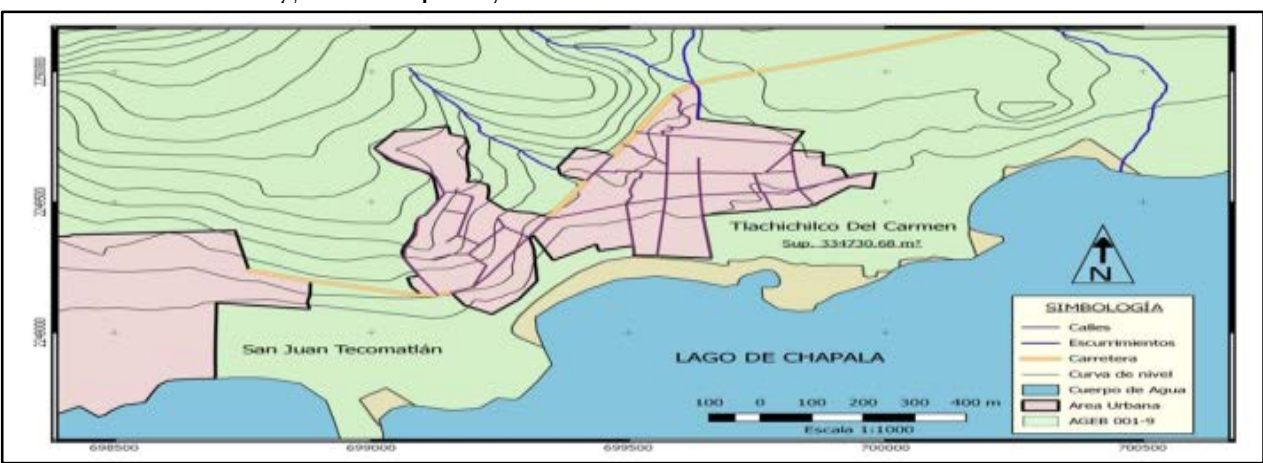


La Localidad cuenta con 437 habitantes, 217 hombres y 220 mujeres, y está considerada con un grado de rezago social muy bajo ${ }^{11}$. Dada su condición de Comunidad Indígena, son reconocidos como comuneros 173 hombres, la mayoría son oriundos y viven en el lugar. De tal manera, los pobladores de Tlachichilco reconocen 2 tipos de autoridades por usos y costumbres: el Agente Municipal, nombrado por el $H$. ayuntamiento de Poncitlán, con una vigencia de 3 años y; la Comisariado de Bienes Comunales, electa por la Asamblea de Comuneros, con una duración en el cargo por el mismo periodo de tiempo.

La localidad de Tlachichilco del Carmen cuenta con una superficie de 750 hectáreas, divididas según el destino de las tierras en:

- Tierras o terrenos para el núcleo de población o asentamiento humano, conformadas por aquellas que están destinadas para la urbanización, los servicios públicos y sociales del lugar,

- Tierras o terrenos parcelados, aquellas designadas a los comuneros para el disfrute del usufructo de las mismas, por lo general destinadas para cultivo agrícola $\mathrm{y}$,

- Tierras o terrenos de uso común, que son las que no están parceladas y son inembargables e imprescriptibles, corresponde sólo a comuneros el derecho de su aprovechamiento para agostadero y terrenos con vocación preferentemente forestal $^{12}$.

Pertenece al núcleo poblacional y terrenos parcelados una superficie de 292 kilometros cuadrados de terrenos de uso común. Las tierras de la comunidad están sujetas a disposiciones relativas al artículo 27 de la Constitución Política de los Estados Unidos Mexicanos, al igual que a la Ley Agraria vigente en México; lo mismo que al Reglamento Interno, parte de los estatutos comunales.

A partir del año 1977 los habitantes del barrio de Tlachichilco inician una serie de trámites legales ante la Secretaría de la Reforma Agraria ${ }^{13}$ del Gobierno Federal o Presidencia de la República en México para que le sea reconocida la titulación de bienes comunales por separado de la Comunidad Indígena de San Juan Tecomatlán. Para tal efecto, el proceso tuvo una larga trayectoria ante Tribunales Agrarios. Pero, en el año 2012 logran el cometido de formar una comunidad independiente y por sentencia del Tribunal Unitario Agrario se adquiere la autonomía legal del territorio; así surge la Comunidad Indígena de Tlachichilco del Carmen o localidad de Tlachichilco del Carmen.

La nueva localidad es considerada una agencia dentro del municipio de Poncitlán, porque cuenta con menos de 1.000 habitantes, por lo que no reúne los requisitos para ser posicionada como Delegación. Así, una vez que la localidad de Tlachichilco del Carmen resuelve la problemática territorial y su autonomía, enfrenta una serie de situaciones sobre uso de recursos de uso común, particularmente la obtención del agua para uso doméstico, como se explica a continuación.

\footnotetext{
11 INEGI 2010.

12 Álvarez, 2006.

13 Denominada a partir del 2012, Secretaría de Desarrollo Agrario, Territorial y Urbano, por sus siglas SEDATU.
}

\section{TERRITORIO PERIURBANO, PAISAJE CODICIADO E INICIO DE LA LUCHA} POR EL AGUA

Antes del año 1984, los habitantes de Tlachichilco se abastecían de agua mediante excavación de pozos en los domicilios. Comentan los pobladores que el manto freático se encontraba a poca profundidad y con facilidad se extraía agua suficiente para uso doméstico. A la vez, por ser colindantes al Lago de Chapala, por usos y costumbres, desde tiempos inmemorables se recurría a él para el baño personal, y el lavado de la ropa ${ }^{14}$. Pero a partir del mismo año se comenzaron a presentar problemas de enfermedades en la piel, en ojos ${ }^{15}$, entre otros padecimientos. De acuerdo a un estudio realizado por la Secretaría de Salud en su momento, la causa fue el grado de contaminantes en el agua del lago.

Lo anterior dio origen a gestionar ante el Gobierno del Estado, la perforación de un pozo profundo y mediante tubería se domiciliara la llegada del agua, y evitar que los pobladores del entonces Barrio de Tlachichilco hicieran uso doméstico del lago. Es así como el suministro del agua potable llegó a los hogares, administrada por la propia comunidad.

En 1992, de manera inesperada por la propia comunidad, personas provenientes de otros países ${ }^{16}$ atraídos por el excelente clima y el bello paisaje, adquirieron terrenos con escrituras fuera de regulación. Construyeron casas residenciales, en su mayoría equipadas con jardines amplios y albercas. Desconociendo los fundamentos y motivos legales, la Comisión Nacional del Agua de la Presidencia de la República en México, otorgó permisos a dichos extranjeros para perforación de varios pozos profundos, reduciendo así la capacidad del manto freático que alimentaba a la población. En razón a tales sucesos, diversos miembros de la comunidad entrevistados externaron que:

“...a partir de que llegaron los gringos nosotros dejamos de tener la suficiente agua en la llave y los pozos de nuestras casas se secaron, antes podíamos usar agua del Lago de Chapala porque estaba limpia, hoy no, ya que huele mal y está verde. El gobierno fue el que les dio permiso a los gringos para que se apropieran del agua, y ahora ellos tienen agua de sobra para sus albercas y sus grandes jardines y nosotros ni para lavar los trastes”.

Esto evidencia el reclamo general y particular de las mujeres, habitantes de la localidad. Por ello, hoy la localidad de Tlachichilco, particularmente, los miembros de la Comunidad Indígena de Tlachichilco del Carmen, señalan que quienes ubican como "los gringos" no, sufren falta de agua para su servicio básico personal y doméstico. Y los pozos construidos antes de 1992 que alimentaban de agua a la población, comienzan a disminuir su afluente, por lo que comienza a evidenciarse lo que hasta hoy en día permanece, una guerra y tensión social por el agua, misma que se distribuye entre 20 y 60 minutos al día en los meses de noviembre a febrero; entre 20 y 30 minutos de febrero a julio. En temporal de lluvia (agosto y octubre) el manto acuífero se recarga y su

\footnotetext{
14 En ese momento el agua del Lago no estaba contaminada, según la percepción de los lugareños.

15 Particularmente conjuntivitis.

${ }^{16}$ Particularmente de Canadá y los Estados Unidos de Norteamérica.
} 
distribución es de tiempo similar que en los periodos antes mencionados.

Desde ese entonces, se tiene la promesa por parte de la Comisión Nacional del Agua, de perforar un nuevo pozo, profundo, para abastecimiento doméstico de la Comunidad Indígena, y solucionar así todo conflicto; pero hasta hoy la promesa no se ha materializado. Es importante señalar que los llamados “extranjeros” no tienen, ni padecen desabasto de agua potable en ningún momento del día, ni en ningún mes de año: aproximadamente sus aproximadamente 30 residencias, cuentan con 4 pozos profundos, mientras que la población de aproximadamente 200 domicilios existe un solo pozo de abastecimiento.

$\mathrm{Al}$ parecer, las instancias gubernamentales no cuentan con capacidades administrativas, financieras y de voluntad política, para implementar sistemas de equidad y distribución del agua en la población de Tlachichilco. El sentir tal tensión social, la precariedad del derecho humano al agua, la injusticia, fue el motivo principal del trabajo de campo aquí expuesto: evidenciar con datos duros el evidente problema ante las autoridades. En su conjunto, es demasiado notorio el despojo del que ha sido objeto la Comunidad Indígena de Tlachichilco, a la que claramente se le niega su respectivo derecho humano al agua.

Hasta el momento presente, la comunidad no ha sido escuchada por las autoridades responsables de solucionar problemas de tal injerencia: instituciones municipales, estatales y federales. Tal tipo de problemas se deben no solamente a las luchas internas de Tlachichilco, sino a la tensión existente entre la apropiación del agua entre espacios urbanos y periurbanos, como se señala en seguida.

\section{AGUA, UNA DISPUTA ENTRE LO} URBANO Y LO PERIURBANO

El agua es un recurso escaso, no renovable, que se degrada a ritmos nunca antes vistos. Algunos países sufrirán más que otros tal destino terrible. Por ello, en el sitio web del organismo World Resources Institute ${ }^{17}$ encargado de medir mediante el Water Stress Index, el estrés hídrico, que va del 0 al 5 , donde 0 representa el menor nivel de escases de agua o low, y 5 el mayor o extremely high, señala que la crisis del agua es un problema mundial que se acelera y naciones como Bahrain, Kuwait, Qatar, San Marino, Singapur, Emiratos Árabes Unidos ${ }^{18}$, Israel, Palestina, Arabia Saudita y Omán, tendrán para el 2040 el mayor nivel de stress hídrico, por lo que se verán en la necesidad de importar el $100 \%$ del agua necesaria para su industria como para la población en general. Pero también naciones,

\footnotetext{
${ }^{17}$ World Resources Institute, 2013.

${ }^{18}$ Fantini, 2003.
}

Fuente: Elaboración propia. particularmente del continente americano, sufrirán el mismo problema, países como Estados Unidos de Norteamérica, México, Haití, República Dominicana, Perú y Chile presentarán entre un 3,18 y 4,45 de dicho estrés, otorgando a México una medición de 3,99, lo que es decir un nivel medium to high de water stress.

Tal problema se suma a los relativos conflictos de disponibilidad y distribución de agua dulce en el mundo: la evidente y progresiva degradación y contaminación del agua actualmente es difícil de revertir. Asimismo, territorios periurbanos, como Tlachichilco, no estarán exentos de dicha problemática. Sufrirán, como ya lo hacen, de un estrés hídrico alarmante, provocado por políticas públicas que privilegian el abastecimiento para ciudades, a pesar de que la dotación del servicio, así como su respectivo saneamiento, debería ser equitativa para todos los espacios, sean urbanos o periurbanos, garantizando el derecho humano al agua en igualdad de condiciones, calidad, cantidad y cobertura.

Los problemas de abastecimiento, consumo y por tanto de accesibilidad, no solamente mantienen en permanente tensión a los espacios urbanos y periurbanos; al interior de estos últimos, también ocurren fenómenos económicos, sociales, culturales y ecológicos que requieren especial atención. Así lo demuestran los resultados aquí expuestos. Evidencian que garantizar el derecho es un desafío que demanda la atención de todos los niveles de gobierno. Y que los retos en materia de agua, no solamente se solucionarán con atender la demanda cada vez más creciente, sino ideando estrategias técnicas, jurídicas, normativas y económicas, para gestionar el servicio con una calidad y continuidad adecuada, abandonando la visión ingenieril que ha predominado por varias décadas.

\section{Mapa 3. Distancia entre Tlachichilco del Carmen y el Área Metropolitana de} Guadalajara

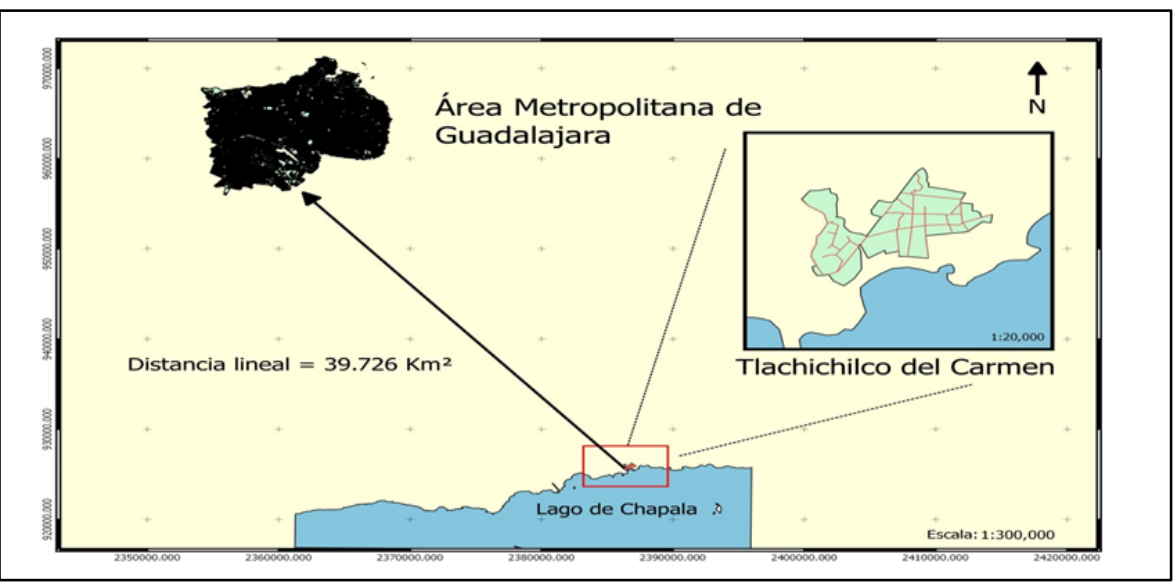

Es necesario el establecimiento de alternativas en gestión con una visión más sustentable. Definir quienes acceden al agua y quienes no, es corresponsabilidad de todos los actores involucrados y con un alto nivel de participación de todos los sectores de la sociedad, no solamente del mercado y del Estado. También será necesario abandonar el viejo reduccionismo que garantiza la dotación de agua a zonas urbanas mientras mantiene a los territorios periurbanos (aquellos que no son urbanos o 
ciudades) en una acelerada decadencia. Tal y como ocurre, se privilegia la dotación de agua extraída de Chapala, para dotar el consumo cada vez más elevado de los habitantes del Área Metropolitana de Guadalajara, sobre el derecho humano de los habitantes de la localidad, irónicamente, localizada al lado de dicho cuerpo de agua. Problemática evidente entre lo urbano y lo periurbano -ver Mapa 3-.

Así, el estudio muestra no solamente el conflicto de demanda por agua entre espacios urbanos versus periurbanos, sino el problema presente en el interior de las propias localidades periurbana en materia de acceso al agua. Y a la par plantea una serie de acciones, alternativas y propuestas que una población periurbana, la de Tlachichilco del Carmen, ha emprendido para al menos en un grado ínfimo, garantizar su subsistencia y por ende su derecho humano al agua, ante la falta de soluciones integrales por parte del municipio de Poncitlán.

DERECHO Humano AL AGUA: ANÁLISIS PRELIMINAR EN UN TERRITORIO PERIURBANO

Es inconcebible que el acceso al agua hoy en día, en pleno siglo XXI, siga bajo el debate internacional para determinarlo como derecho humano o como mercancía, es decir, bajo una pugna entre mercado y sociedad. Al respecto, países como México, han optado por reconocer desde el 2012 en su Ley Suprema ${ }^{19}$, que:

“...toda persona tiene derecho al acceso, disposición y saneamiento de agua para consumo personal y doméstico en forma suficiente, salubre, aceptable y asequible.”

Atribuyendo al Estado la obligación que tiene por garantizar este derecho, para que este defina en sus marcos normativos las bases, apoyos y modalidades para su realización, en forma equitativa y sustentable, mediante mecanismos de participación entre los niveles de gobierno Federación, de las entidades federativas y de sus municipios, con la participación corresponsable la ciudadanía para la consecución de dichos fines.

Sin embargo, la idea anterior dista mucho de la realidad. El derecho humano que protege el artículo 4 de la Constitución Política de los Estados Unidos Mexicanos, señala que todo individuo que habite dentro del territorio de esa nación gozará de agua en calidad y cantidad suficiente $\mathrm{y}$ asequible para satisfacer sus necesidades básicas, sea suministrado por el Estado, o por particulares o de forma mixta. Tal artículo, no es respetado en todo el territorio nacional.

Así, localidades como Tlachichilco del Carmen, se encuentran en un estado contrario a lo que debería ser. El servicio del agua, considerado primario, obligatorio, domiciliario, abierto y esencial, ya que tiende a satisfacer necesidades relacionadas con la existencia humana, al que cualquiera debería acceder ${ }^{20}$, es vulnerado por la inoperante actividad del Estado en todos sus niveles de gobierno, al igual que por dos grandes conjuntos del Área Metropolitana de Guadalajara y la de canadienses y norteamericanos residentes en México.

Considerar el acceso al agua como un derecho humano, encuentra sus bases en que su prestación es

\footnotetext{
19 Jalomo, 2011.

${ }^{20}$ Ayala, 1996.
}

vital, irrenunciable e indispensable para la vida, por lo que debería prestarse a través de un sistema de redes a todos y para todos ${ }^{21}$, satisfaciendo así necesidades colectivas a la vez que individuales, mediante mecanismos institucionales, sin exclusión; todos deben contar con la misma accesibilidad en cantidad y calidad, en derechos y obligaciones, estableciendo por ejemplo tarifas equitativas a miembros de un mismo grupo de usuarios.

Accesibilidad, entendida como captación, distribución, disponibilidad, asequibilidad, calidad, obligatoriedad, adaptabilidad, continuidad, no discriminación, abastecimiento y uso, en Tlachichilco del Carmen su significado no es tal. Por tal, en una segunda etapa de la investigación, se reparará en desecho, alcantarillado, cloaca, saneamiento y disposición final de las aguas residuales ${ }^{22}$.

¿Qué comprende tal derecho en términos formales? Comprende la distribución de agua apta para consumo humano, incluida su conexión y medición. Abarca además actividades complementarías de capación de agua y su procesamiento, tratamiento, almacenamiento, conducción $^{23}$ y transporte. No tener acceso al recurso, por ende al servicio que lo materializa, violenta no solamente un precepto constitucional, sino todas aquellas garantías fundamentales como el derecho al trabajo que utiliza agua para producción industrial; para la generación de energía; producción agropecuaria; alimentación; cocina; aseo personal; salud; etc.

El derecho a gozar de un medio ambiente sano y digno para el desarrollo, servicios ecológicos que proveen plantas, árboles y que constituyen garantías, en Tlachichilco es violentado. Las necesidades básicas de los comuneros, no son atendidas; el servicio con que cuentan no atiende al interés general y sólo se favorecen intereses particulares, las tarifas no inducen a la mejor utilización de los recursos disponibles y del servicio en sí.

Así, principios circunscriptos al derecho humano al agua, como igualdad, equidad social, viabilidad financiera, estabilidad, sencillez y calidad, no aplican en pobladores originarios de Tlachichilco del Carmen. Es decir, se violentan los 5 ejes que fundamentan tal derecho: disponibilidad, accesibilidad, asequibilidad, calidad y la no discriminación ${ }^{24}$.

Consecuentemente, tres aspectos cobran vital importancia: la disponibilidad, la distribución y la calidad. El primero hace referencia a una cualidad, condición o situación respecto de un bien o cosa disponible libremente para usarse o utilizarse, estando al alcance o no; el segundo refiere a la distribución dentro de un territorio; el último, se relaciona con las propiedades químicas y físicas del recurso, porque no es lo mismo tener a la mano agua con elevados niveles de contaminación, que agua potable libre de metales pesados y otros contaminantes perjudiciales para la salud humana. Elementos como disponibilidad y calidad, refieren al agua como un recurso escaso a nivel planetario; su distribución es inequitativa y es un recurso bastante

\footnotetext{
${ }^{21}$ Idem, 1996, 43-51.

22 Ibidem, 1996.

23 Ayala, 1996, 54

24 Carbonell, 2004 y Jalomo, 2011.
} 
vulnerable; su accesibilidad no es igual en todas partes y no se tienen cantidades proporcionales que satisfagan la demanda creciente de la población.

Se entiende por disponibilidad la cantidad a que tiene derecho todo individuo o habitante por día, para uso, goce y disfrute que de conformidad con la Organización de Naciones Unidas, se promedia en 50 litros diarios de agua potable por habitante ${ }^{25}$ para cubrir necesidades mínimas básicas de alimento y aseo, más 100 litros para satisfacer las necesidades generales ${ }^{26}$, es decir, 150 litros por habitante al día. Por accesibilidad, se entiende, no en términos totalizantes sino en términos particulares, la distancia en que se encuentra el recurso para ser aprovechado. Por asequibilidad, al coste del agua. Por calidad, al grado de pureza del recurso en términos físicos y químicos que la vuelven o no apta para consumo humano. Y finalmente por no discriminación, a las condiciones sociales, económicas, políticas y culturales que generan un inequitativo reparto del recurso. Un reparto injusto en Tlachichilco del Carmen.

Figura 1. Muestra para el estudio

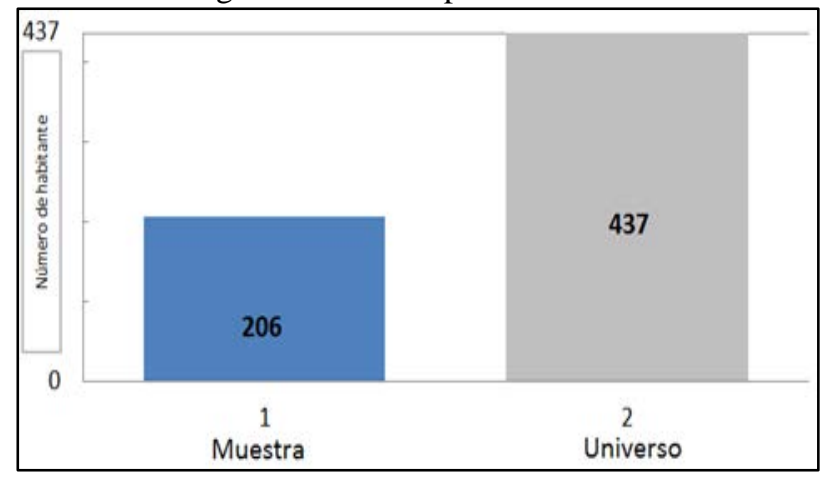

Fuente: Elaboración propia con base en trabajo de campo realizado en la localidad de Tlachichilco del Carmen en el 2016.

A continuación se muestran y analizan los datos donde se evidencia que en Tlachichilco del Carmen, Jalisco, ocurre un problema de acceso equitativo al agua al interior de un territorio periurbano que impide lograr un desarrollo sustentable en pleno siglo XXI.

Figura 2. Personas originarias de Tlachichilco del Carmen

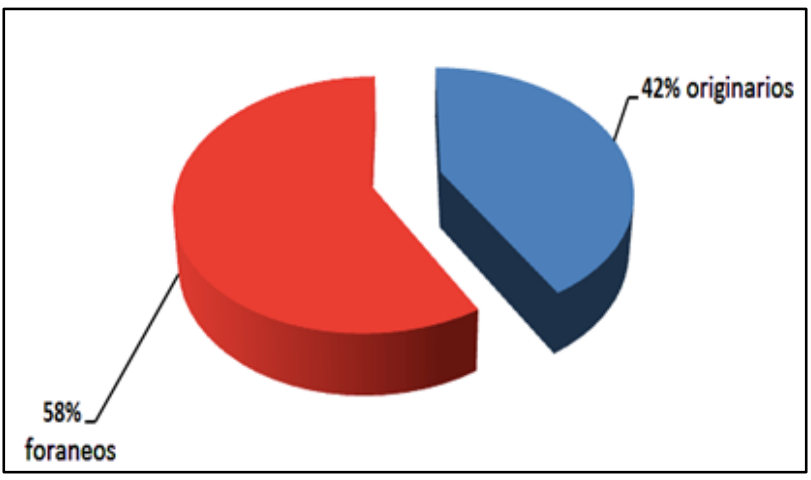

Fuente: Elaboración propia con base en trabajo de campo realizado en la localidad de Tlachichilco del Carmen en el 2016.

Del total de 206 personas encuestadas -ver Figura 1-, $58 \%$ nacieron en territorios distintos al de Tlachichilco del Carmen; un 42\% son originarios de dicha comunidad,

\footnotetext{
${ }^{25}$ Bartram, 2002. Gleick, 1996

${ }^{26}$ FNUAP, 2001.
}

pero el $100 \%$ de los sujetos estudiados tienen aproximadamente viviendo en Tlachichilco 45 años, por lo que conocen de primera mano el problema en dicho territorio periurbano respecto al acceso al agua -ver Figura 2-.

Así, cuando se habló sobre disponibilidad con los sujetos, manifestaron que tienen en promedio 36 minutos al día agua en sus domicilios, lo que da una distribución por habitante al día de entre 6 y 7 minutos para cada persona -ver Figura 3-. Información que si se distribuye a lo largo del año, demuestra que solamente disponen de ese tiempo de agua 11 meses de los 12 que abarcan un año, bajo la cantidad de minutos por día que antes ya se enunció. Ya que por ejemplo el $100 \%$ de los sujetos encuestados señalan que en el mes de mayo solamente tienen agua 7 días dentro del mes.

Figura 3. Minutos al día con agua potable

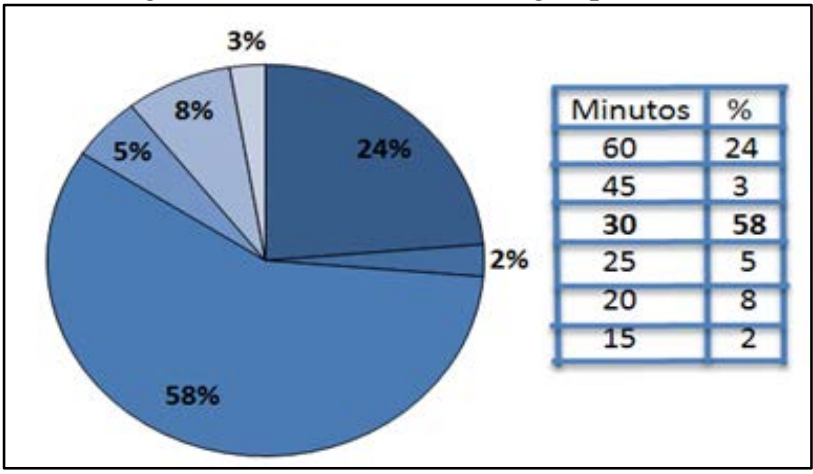

Fuente: Elaboración propia con base en trabajo de campo realizado en la localidad de Tlachichilco del Carmen en el 2016.

Cuando se cuestionó respecto a cuántos litros de agua potable recibían por persona/hogar durante un día, la cifra es aún más alarmante, disponen de solo 31 litros por individuo. Por lo que el $100 \%$ de las personas refieren que la dotación de agua que reciben diariamente es insuficiente para cubrir sus necesidades básicas -ver figura 4-, considerando que al menos deberían tener un promedio diario de 105 litros por habitante al día para satisfacer sus necesidades personales y domésticas.

Durante el levantamiento de información, fue posible percatarse de que 4 de 38 hogares contaban con pozos propios, de los cuales, los habitantes de los 4 domicilios, señalaron que el manto freático había bajado a tal grado que no los utilizaban. Ello coincidió con la llegada de los “gringos” y la creación de pozos en sus casas.

Figura 4. ¿El agua que recibe es suficiente para cubrir sus necesidades diarias?

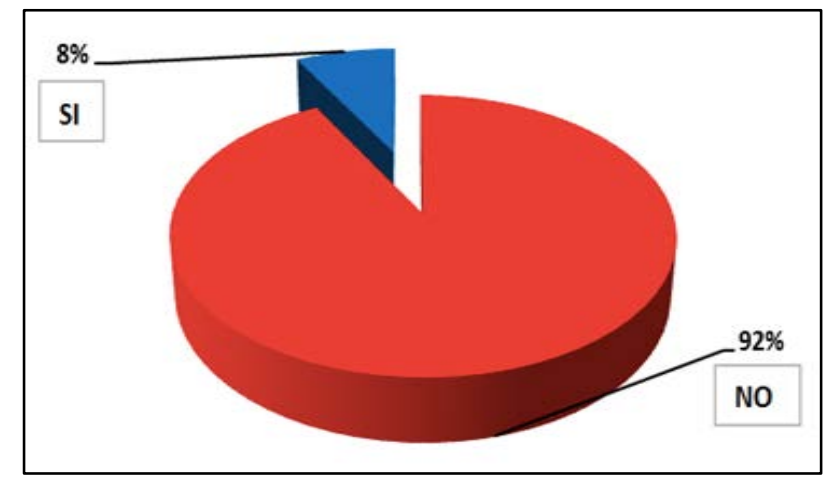

Fuente: Elaboración propia con base en trabajo de campo realizado en la localidad de Tlachichilco del Carmen en el 2016. 
Representó un descubrimiento asombroso, conocer el hecho de que como parte de las acciones voluntarias, que los pobladores de Tlachichilco implementaban para cubrir sus necesidades de agua, acciones representativas de una Nueva Cultura del Agua: ahorro, reúso, compartir con sus vecinos más necesitados e incluso captando agua de lluvia. Tal y como ocurre obligatoriamente en legislaciones como la de Cataluña, donde existen 32 municipios que han aprobado Ordenanzas de Ahorro del Agua, formando una Red de Municipios por la Nueva Cultura del Agua, con el objeto de regular la incorporación y la utilización de sistemas de ahorro, como también adecuar la calidad del líquido al uso que se haga en los edificios, otras construcciones y actividades, y de determinar en qué casos y circunstancias será obligatoria $^{27}$.

Bajo esta filosofía de Nueva Cultura del Agua, 63\% de los domicilios encuestados, más de la mitad de la población, manifestaron que captan y usan el agua de lluvia con métodos rudimentales: colocar baldes, cubetas, tambos, cazuelas y otros contenedores al momento de la lluvia, para allegarse de una mayor cantidad ante el desabasto que sufren -ver Figura 5-.

Respecto a accesibilidad como termino particular, no globalizante, es posible mencionar que pese a que en ocasiones se necesita caminar algunos metros para ir por agua, por ejemplo, con sus vecinos, el 100\% de los encuestados manifestó que recibe el líquido directamente en sus casas y cuando escasea en la red de distribución que llega hasta sus domicilios, tardan 38 minutos en ir y conseguir a otros lugares; para ello deben trasladarse alrededor de 38 metros; ambas cifras distribuidas por habitante al día. Ello evidencia aspectos de accesibilidad, que implica a su vez ciertos retos: en las encuestas un individuo manifestó estar afectado de sus piernas, lo cual le impide salir a buscar agua con sus vecinos.

Figura 5. Aprovechamiento de agua de lluvia.

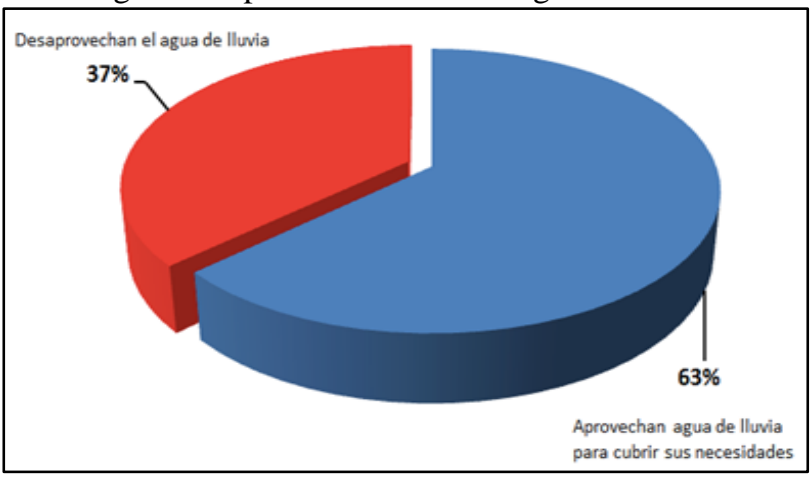

Fuente: Elaboración propia con base en trabajo de campo realizado en la localidad de Tlachichilco del Carmen en el 2016.

Al preguntar a los encuestados quién de la comunidad era encargado de realizar el cobro del agua, la mayoría manifestó que es el señor Salvador Betancourt Chávez a quien todos ubican como Agente Municipal. Por lo general le pagan cada mes de enero, una cantidad de \$851 pesos anualmente (aproximadamente 42,55 dólares americanos), sin que aprecien la resolución al desabasto. El incremento del coste en comparación con años anteriores, hace posible medir aspectos de asequibilidad.
Aunque es oportuno señalar que solamente $58 \%$ de los hogares encuestados, reconocieron estar al corriente en el pago del servicio, el restante $42 \%$ no lo están por el pésimo suministro y la falta de atención por parte del Municipio de Poncitlán. Hogares que estaban al corriente del pago, de los que un 53\% están al corriente en su pago y por ende obtiene algún tipo de descuento en el coste anual. Como lo reciben también los mayores de 60 años, jubilados o pensionados. Respecto a no discriminación, se logró encontrar una marcada división entre quienes opinaban que no todas las personas en la localidad pagaban el mismo precio por el agua, 50\% y quienes señalaban lo contrario, el otro $50 \%$ restante -ver Figura 6-.

Figura 6. ¿Todas las personas en Tlachichilco pagan el mismo precio por el agua potable?

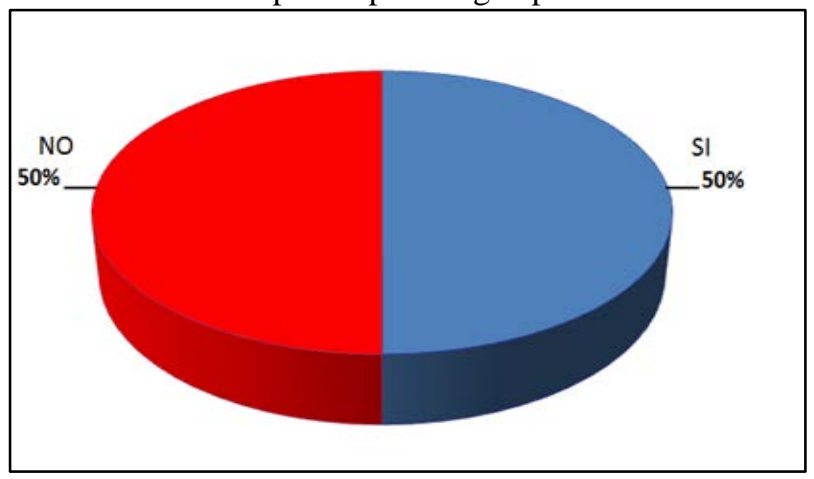

Fuente: Elaboración propia con base en trabajo de campo realizado en la localidad de Tlachichilco del Carmen en el 2016.

Referente a otro aspecto que pretendía medir la no discriminación, cuestionando si todas las personas en la localidad recibían la misma cantidad de agua potable, la diferencia fue abismal y rotunda: el 18\% manifestó que sí, mientras que el $82 \%$ restante señalaron que existen favoritismo, prelación y discriminación en cuanto a la dotación de agua por domicilio y por habitante al día -ver Figura 7-. Siendo los usos que se le dan al agua mayoritariamente destinados a necesidades básicas, como lavar el baño, la ropa, limpiar la casa, ducharse y escasamente para regar plantas y bañar mascotas en casa. Todos los encuestados aseguran que les es imposible, por la escasa agua que reciben, darse el lujo de lavar el coche, tener albercas o piscinas o regar jardines, tal como hacen "los gringos" que no sufren por desabasto.

Figura 7. ¿Todas las personas en Tlachichilco reciben la misma cantidad de agua potable?

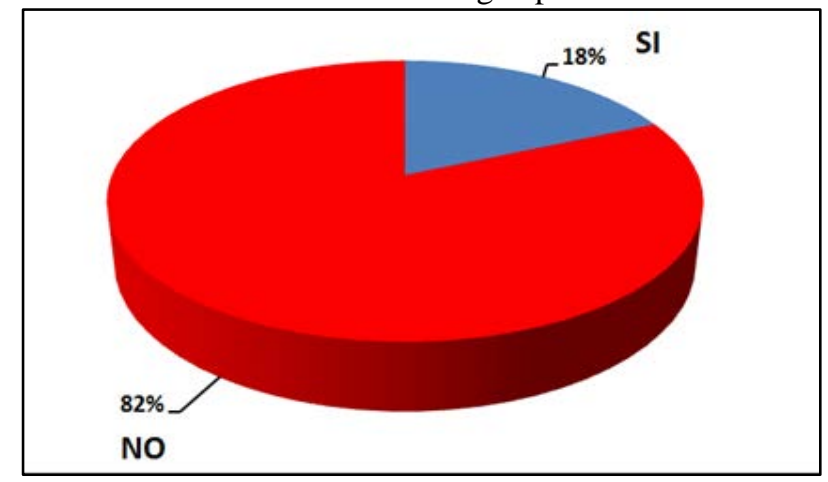

Fuente: Elaboración propia con base en trabajo de campo realizado en la localidad de Tlachichilco del Carmen en el 2016.

\footnotetext{
${ }^{27}$ Meseguer, 2009, 271.
} 
Finalmente, en calidad de la poca agua recibida, un $100 \%$ de los encuestados manifestó que ninguna persona se ha enfermado por usar este recurso que les llega de la red como ya se evidenció, escasamente los suministra y deja en estado de vulnerabilidad cuando se habla del derecho humano al que hoy aspiran.

Asimismo, entre algunas observaciones relevantes, los encuestados manifestaron que ya están acostumbrados, a tener dos veces por semana problemas serios de abasto de agua, pues el pozo que abastece a la comunidad se queda con poca agua y en temporada de secas el problema se agrava, entre mayo y junio.

Incluso fue posible descubrir con este estudio unas personas cercanas al Presidente Municipal de Poncitlán tienen una bomba pegada al tanque que abastece a la comunidad $^{28}$ y por ende reciben más agua que todos los otros comuneros. Y los extranjeros tienen suficientes pozos propios por lo que no padecen de escasez de agua, el agua huele mucho a cloro, que algunos comuneros se ven en la forzosa necesidad de comprar incluso agua a los estadounidenses como consecuencia del desabasto del que son objeto.

Además, debido a la carencia de agua las plantas y hortalizas de los comuneros se han secado y como el agua se distribuye por gravedad algunas casas reciben más que otras y el señor Betancourt sabe que existen fugaz en la red de distribución y no hace nada por solucionarlas.

Sobre estas afirmaciones el señor Rigoberto Betancourt, Agente Municipal de Poncitlán, Jalisco 2013-2015 señaló que:

“...debido a la influencia de una persona que necesitaba un favor, se hicieron las perforaciones con el permiso de la comunidad, y existen de 3 a 4 pozos en casas de los extranjeros, pozos que deberían de ser de uso para toda la comunidad pero se hace uso particular solamente. Por lo que se realizó una junta en 2015, para solicitar a los extranjeros que cedieran los pozos al Ayuntamiento pero estos no quisieron cederlos por lo que existen varios procesos legales entre ellos y los comuneros, que aún están en curso en el Tribunal Agrario. Y los extranjeros quieren favores o ayuda para sus terrenos por parte de la comunidad, pero no quieren acceder ni llegar a acuerdo que solucionen el problema del desbasto de agua. E incluso, cuando la comunidad ha tenido problemas con el abastecimiento del agua, los extranjeros con los pozos a nombre de la comunidad dentro de sus propiedades les han llegado a vender el agua por metro cúbico durante un mes aproximado, más caro que como lo cobra el propio organismo denominado SIAPA en el Área Metropolitana de Guadalajara”.

Son 40 extranjeros aproximadamente quienes se abastecen de los pozos construidos en sus propiedades. Y alrededor de 500 habitantes, de los cuales quedan 140 comuneros originarios, sufren el desabasto. Pero los extranjeros no quieren llegar a ningún acuerdo; al contrario, menosprecian a los habitantes y hasta los ofenden. Por todo el problema de tierras adquiridas de forma irregular, un abogado lleva el caso contra los extranjeros, pero desconoce el avance del juicio a la fecha.

"Los extranjeros han hecho muchas cosas que han ocasionado gastos para la comunidad, por lo que los comuneros han querido orientarse para saber cuál será la solución para el problema pero no les dan mucho apoyo y siguen pagando aproximadamente 800 pesos cada domicilio por un servicio que es escaso y deficiente".

Por su parte, el señor Salvador Betancourt, hermano del señor Rigoberto, en entrevista refirió que además de ser comunero de la Comunidad, él es el Auxiliar de Bombas en la localidad, designado así por los Presidentes Municipales, Víctor Carrillo y Juan Carlos Montes, en sus diferentes periodos de gobierno, por lo que ya lleva en dicho encargo 4 años, tres de la administración pasada y uno de la actual. Recibiendo un sueldo mensual de 1,900 por cobrar el agua y dar mantenimiento al sistema que dota la poca agua que se otorga a los habitantes; es parte de sus funciones también prender la bomba en la mañana.

Corrobora que, efectivamente, la mitad de la población no está al corriente de los pagos por el agua que reciben. Y que los “extranjeros” no pagan por el agua recibida, porque ellos están organizados de forma independiente. Refiere además que los aumentos en el costo del agua para los comuneros, responde a órdenes de la propia Presidencia de Poncitlán y que todos deberían pagar a finales o principios de cada año, pago contra el que él entrega un recibo a cada usuario, mismos que pagan en efectivo.

Constata que, efectivamente, en mayo el agua se escasea aún más, pero que ya se trabaja perforando un nuevo pozo por parte del Municipio; desconoce los gastos de dicha obra. Señala desconocer qué ocurre con el dinero recaudado por pago de agua. Algunas personas de la comunidad reciben más agua y a otros menos y que todos reciben por lo general el agua en sus domicilios, poca, pero reciben. Indica el señor Betancourt que quienes se encargan de clorar el agua vienen directamente de Poncitlán una vez por semana, por ello él no puede hacer nada para remediar el exagerado olor a cloro. Constata que, aproximadamente 10 casas nunca han pagado por el servicio, se niegan a pagar, simplemente. Lo que repercute en el monto recaudado y por ello no todos pagan igual por el servicio en la comunidad.

Aparte valida que, nadie se ha enfermado últimamente por tomar el agua de la llave, ya que existe una potabilizadora en la comunidad que distribuye con costo independiente a la población, agua de garrafón a todos los habitantes. Y que incluso cada 2 años, acude personal del Municipio de Poncitlán para muestrear el agua y corroborar que no contenga agentes infecciosos y salvaguardar la salud de la población.

\section{CONCLUSIONES}

Queda demostrado que hay una mayor cantidad de personas que acceden, al agua potable; que la principal actividad que realizan con el agua la mayoría de los comuneros es para atender necesidades básicas para su subsistencia y que la cantidad, asequibilidad, duración y/o disponibilidad que tienen al agua potable por hogar en la localidad vulnera el derecho humano al agua consagrado en la Carta Magna de México y por consiguiente deja en estado de indefensión a habitantes dentro del territorio periurbano frente a otros actores como por ejemplo los “extranjeros”, a los que algunos de

\footnotetext{
${ }^{28}$ A la que refieren como los hermanos Betancourt.
} 
ellos refieren como “invasores”, por la ocupación irregular de tierras que hicieron dentro de la localidad.

Además, es posible señalar que Tlachichilco, es ejemplo a nivel país de las acciones dirigidas a atenuar el déficit de agua, aprovechando los $449 \mathrm{~km}^{3}{ }^{29}$ de agua totales que se precipitan cada año en México. Y que aunque este ejemplo es poco representativo de la cultura que existe por captar y aprovechar el agua de lluvia dentro del país, representa una buena práctica que podría ser tomada en cuenta a lo largo del territorio nacional. Es importante también manifestar que los comuneros de Tlachichilco permanecen muy por debajo de los 329.496 litros de agua apta para consumo humano por habitante al día, empleados en otras partes de México. Incluso su consumo, disponibilidad y dotación, es más bajo del promedio estimado de agua por habitante al día en México (252 litros), cifra que no alcanza lo indispensable (150 litros, recomendados por la Organización de las Naciones Unidas) ${ }^{30}$.

Falta entonces mucho trabajo en la comunidad para que el Estado garantice el derecho humano al agua, regulando y solucionando el problema de acceso a un bien de carácter difuso o colectivo. A raíz de las reformas constitucionales antes explicadas, en México, la propiedad del agua debería responder a una protección de corte social versus lo puramente económico que atribuye al agua una noción de mercancía susceptible de comercialización, a la cual solamente puede acceder quienes cuentan con la posibilidad de adquirirla. Por ello, en México debería observarse el agua como un bien de dominio público y social, al que todos tienen derecho a acceder en igualdad de condiciones, calidad y equidad.

Así, bienes públicos llamados también recursos de propiedad pública, como el agua, se identifican por tres características: la no exclusión, el coste proporcional nulo para consumidores adicionales y la no rivalidad, condiciones todas no respetadas en Tlachichilco. Adicionalmente, para que un recurso sea considerado bien público, debe cumplir dos condiciones: contar con libre acceso para cualquiera quien desee usarlo y existir algún tipo de interacción adversa entre usuarios. De poderse limitar el acceso a un bien público, también podría gestionarse el recurso por aquellos que pudieran imponer dicha limitación y, por otro lado, si no existiera una interacción adversa entre los usuarios, no habría razón para imponer la limitación.

En una visión alternativa, también se hace referencia a bienes públicos cuando se refiere a derecho de propiedad en recursos en los que todos los propietarios poseen el mismo derecho para su uso. Por lo tanto, significa que tal derecho no se pierde si el propietario no lo usa, pero también significa que no todos los propietarios utilizarán siempre la misma cantidad del recurso a lo largo del tiempo. Así, los potenciales usuarios que no son miembros, están excluidos del uso del mismo. Por lo que el concepto de propiedad implica la potestad implícita de exclusión de todos aquellos que no son propietarios. Así, es posible distinguir entre propiedad común, propiedad de todos y propiedad pública o bien público, este último caracterizado por ser de libre acceso.

\footnotetext{
29 SEMARNAT, 2012.

30 Jalomo, 2011.
}

Para que la propiedad de un recurso sea operativa, es imprescindible la existencia de reglas claras y la existencia de un conjunto de arreglos institucionales o acuerdo entre propietarios, a lo que se le puede denominar la institución de la propiedad común. Tal carácter de bien público atribuido al agua, hace necesario que para el reconocimiento de dicho derecho, se establezcan formas de satisfacción institucionales, que permitan garantizar a todos aquellos derechosos del agua, la posibilidad para reclamar acceso al servicio, siendo o no del mismo al momento del reclamo.

Sobre todo, cuando potencias extranjeras, por ejemplo Estados Unidos y refresqueras como Coca-Cola, se apropia de enormes reservas de agua, adquiriéndolas como producto circulante en el mercado, almacenándolas e incluso resguardándolas; un hecho cada vez más evidente y racional, que auguran futuras guerras no por petróleo, sino por agua ${ }^{31}$. Síntomas de ello: los problemas suscitados entre la Ciudad de México y el Estado de México durante décadas, por citar el caso de un conflicto al interior de México que manifiesta está clara tensión por el agua $^{32}$, tensión a la que se está sumando la localidad de Tlachichilco del Carmen.

No cabe duda que la judicialización de tal derecho, permitirá acceder a medios de solución de conflictos que se susciten entre concesionarios, inversores, usuarios y no usuarios. Judicialización en puerta para el caso que nos ocupa, y que abrirá mecanismos a través de observadores internacionales, como es el caso del Tribunal Latinoamericano del Agua, al que varias comunidades organizadas mexicanas han acudido en contra de acciones sobre todo del gobierno, que tienden a afectar a sus comunidades con la construcción de megaproyectos hídricos. Y que de forma interna ha venido atendiendo las diferentes comisiones de derechos humanos en el país ${ }^{33}$.

Tal protección y judicialización del derecho al agua, debería encontrar eco en ejemplos donde se han instaurado mecanismos de protección para velar por el mismo, como el de la Defensoría del Agua y los Derechos Humanos ${ }^{34}$, que funciona actualmente en Mendoza, Argentina ${ }^{35}$. En México, la protección del derecho no es un asunto ajeno; los problemas por parte de los ciudadanos en relación a acceso al agua potable y a saneamiento, constituye otro de los pendientes que enfrentan todos los niveles de gobierno en todo el territorio. Más cuando se trata de la localidad aquí estudiada, caso que permite corroborar la necesidad de incluir la participación social en toma de decisiones y corresponsabilidad gobierno-sociedad, mediante estrategias como la de la Nueva Cultura del Agua ${ }^{36}$.

Mecanismos, que pretenden permitir la creación de un sistema de gestión sostenible de los recursos disponibles, que satisfagan los consumos de la población -urbana, industrial y agrícola, sin deteriorar los ecosistemas acuáticos. A su vez que facilitarán transitar del modelo clásico de gestión del agua, el de la oferta y el del control

\footnotetext{
${ }^{31}$ Welzer, 2010.

32 Perló, 2009.

33 Ibidem, 2006 y 2009

34 Por sus siglas DAyDH.

35 Jalomo, 2011.

36 Meseguer, 2009, 263.
} 
de la demanda, por opciones socialmente y ecológicamente responsables, que se aproximen más a una política basada en la sostenibilidad, que a la política hidráulica tradicional de la oferta y la demanda. Esta última relacionada a la palabra políticamente maldita, trasvase $^{37}$.

Problemas como los de Tlachichilco, persistirán de no cambiar el modelo de desarrollo de gestión del agua que impide transitar a la sustentabilidad que permita garantizar el derecho humano al agua ${ }^{38}$. El derecho al acceso, tendría que plantearse a manera que estimule a los gobiernos a considerar en primer lugar a los pobres, a los vulnerables y marginalizados; a la par, cumplir con una base de racionalización y uso sustentable, que garantice un acceso equitativo y no sólo en zonas metropolitanas. Como señalaba Gandhi, cuanto más indefensa es una criatura, más derecho tiene a ser protegida por el hombre, contra la crueldad del hombre. Cumpliendo con los principios básicos de dicho derecho, lograr la equidad social, la viabilidad financiera, la estabilidad, la sencillez y la calidad, se asegura la concordia y el bien común social. Por consiguiente, será oportuno continuar realizando estudios como el presente, porque el tema más que actual es relevante y necesario. Falta estudiar cómo se gestiona el agua en otras comunidades; cómo se problematiza la escases de agua en relación a procesos de apropiación del territorio. Otras posibilidades serían las capacidades de habitantes en autogestión; las distintas formas de acceso al agua a nivel local; problemas generados respecto a distribución. Tales estudios permitirían un acercamiento regional y territorial al servicio público del agua.
También es innegable que se requieren investigaciones no solamente generalizadoras, que den cuenta del fenómeno a nivel macro social, sino otras que como la aquí presentada, reconozcan la importancia del análisis minucioso de particularidades existentes relacionadas a la ausencia de respeto al derecho humano al agua en distintas comunidades $y$, por supuesto, la desigualdad de acceso existente.

\footnotetext{
${ }^{37}$ Gómez, 2012. Prat, 2009.

38 Jalomo, 2011.
} 


\section{BIBLIOGRAFÍA}

Allen A. 1994: "Re-assessing urban development: Towards indica- tors of Sustainable Development at urban level”, en VV. AA.: Ensayo sobre las tres esferas, Working Paper DPU -Development Planning Unit-, UCL, 1 , https://www.jstor.org/stable/4029445?seq=1\#metadata_info_tab_contents

Álvarez Icaza Longoria, P. 2006: "Los recursos de uso común en México: un acercamiento conceptual”, Gaceta Ecológica, 80, 5-17.

Ayala Caldas, J. E. 1996: Elementos teóricos de los servicios públicos domiciliarios. Santafé de Bogotá, D.C. (Colombia), Ediciones Doctrina y Ley.

Bartram, J. and Howard, G. 2002: Domestic water quantity, service level and health: what should be the goal for water and health sectors, Switzerland, OMS.

Baudrillard, J. 1970 [2009]: La sociedad de consumo: sus mitos, sus estructuras, Madrid, Editorial Siglo XXI.

Boehm, B. 2013: Agua, tecnología y sociedad en la Cuenca LermaChapala. Una historia regional Global. México,: Nueva Antropología.

Carbonell, M. 2004: "Notas para el estudio del derecho a la alimentación y el derecho al agua”, en Biblioteca Jurídica Virtual del Instituto de Investigaciones Jurídicas de la Universidad Nacional Autónoma de México $\quad$-UNAM-, $\quad 1, \quad 11-21, \quad$ https://revistascolaboracion.juridicas.unam.mx/index.php/rev-facultad-derechomx/article/viewFile/28666/25917

Fantini, E. 2003: Geopolitica dell'acqua, seminario al corso di Geografia Politica, Roma, Italia, Academia University Press.

Fernández García, Fernando. 2000: "Iniciativa de Reforma a la Ley General de Asentamientos Humanos", en Revista del Centro de Estudios Sociales y de Opinión Pública -CESOP- de la Cámara de Diputados del Congreso de la Unión, 68, 1-32, file:///C:/Users/DoctoGeo/Downloads/Agenda_Desarrollo_Metropolitano_Doct 068.pdf.

FNUAP. 2001: El estado de la población mundial 2001, Huellas e hitos: población y cambio del medio ambiente, New York, Editorial Fondo de Población de las Naciones Unidas.

García García, A y Martínez Quezada, J. A. 2005: “Agua doméstica en San
Cristóbal de Las Casas, Chiapas: entre la gestión local, la centralización y la privatización (1935-2004)”, Revista de Geografía Agrícola, 35, 135-149.

Gleick, P. H. 1996: "Basic water requirements for human activities: meeting basic needs”, Water International, 21, 83-92, https://doi.org/10.1080/02508069608686494.

Gómez Fuentes, A. C. 2012: Territorio y resistencia social. La lucha en contra de la construcción de presas y trasvases (Aragón, 1985-2010). Guadalajara (México), El Colegio de Jalisco.

Jalomo Aguirre, F. y Martí Capitanachi, D. R. 2016: “Taxonomía de modelos de gestión urbana del agua: experiencias de diversas latitudes y conclusiones aplicables al caso mexicano", Revista Tecnogestión: Una mirada al ambiente, 13(1), 76-91.

Jalomo Aguirre, F. 2011: “Gobernar el territorio entre descentralización y metropolización: el Patronato y el SIAPA como formas de gestión, 1952-2006 y escenarios prospectivos”. Guadalajara (México), Universidad de Guadalajara

Meseguer Penalva, M. J. 2009: "Agua y vivienda bioclimática", en Montoro Chiner, M. D. J. (coords.): El agua: estudios interdisciplinares. Barcelona Institut de l' Aigua y Atelier, Libros Jurídicos, 259-281.

Perló Cohen, M. 2009: "La derrota de las aguas", Nexos, 382, 34-36.

Prat, N. 2009: "30 años de gestión del agua en Catalunya. De la oferta ilimitada a la sostenibilidad ambiental", en Montoro Chiner, María de Jesús (coord.): El agua: estudios interdisciplinares. Barcelona Institut de l' Aigua y Atelier, Libros Jurídicos, 207-229.

SEMARNAT. 2012: Informe de la situación del medio ambiente en México, Compendio de estadísticas ambientales, indicadores clave y desempeño ambiental, México D.F, Editorial de la Secretaria de Medio Ambiente y Recursos Naturales.

Suárez, Mario. 2004: Interaprendizaje holístico de matemáticas. Ibarra (Ecuador), Editorial Gráficas Planeta.

Vattimo, G. 1981: “Al di là del soggetto”, Milán, (Italia) Feltrinelli 